\title{
A Second-Order Boundary Value Problem with Nonlinear and Mixed Boundary Conditions: Existence, Uniqueness, and Approximation
}

\author{
Zheyan Zhou and Jianhe Shen \\ School of Mathematics and Computer Science, Fujian Normal University, 350007 Fuzhou, China \\ Correspondence should be addressed to Jianhe Shen, jhshen@fjnu.edu.cn
}

Received 31 December 2009; Revised 14 April 2010; Accepted 3 July 2010

Academic Editor: Paul Eloe

Copyright (c) 2010 Z. Zhou and J. Shen. This is an open access article distributed under the Creative Commons Attribution License, which permits unrestricted use, distribution, and reproduction in any medium, provided the original work is properly cited.

A second-order boundary value problem with nonlinear and mixed two-point boundary conditions is considered, $L x=f\left(t, x, x^{\prime}\right), t \in(a, b), g\left(x(a), x(b), x^{\prime}(a), x^{\prime}(b)\right)=0, x(b)=$ $x(a)$ in which $L$ is a formally self-adjoint second-order differential operator. Under appropriate assumptions on $L, f$, and $g$, existence and uniqueness of solutions is established by the method of upper and lower solutions and Leray-Schauder degree theory. The general quasilinearization method is then applied to this problem. Two monotone sequences converging quadratically to the unique solution are constructed.

\section{Introduction}

The investigation of boundary value problems (denoted as BVPs for short) of ordinary differential equations is of great significance. On one hand, it makes a great impact on the studies of partial differential equations [1]. On the other hand, BVPs of ordinary differential equations can be used to describe a large number of mechanical, physical, biological, and chemical phenomena; see [2-5] for example. So far a lot of work has been carried out, including second-order, third-order, and higher-order BVPs with various boundary conditions.

As far as we know, for a long term most of works focused on existence and uniqueness of solutions. The works relating to approximation of solutions are relatively rare. In recent years, some approximate methods, such as the shooting method [6], monotone iterative technique [7], homotopy analysis method [8], and general quasilinearization method have been applied to BVPs for obtaining approximations of solutions. Among these methods, the general quasilinearization method becomes more and more popular.

The quasilinearization method was originally proposed by Bellman and Kalaba [9]. It is a very powerful approximation technique and unlike perturbation methods, is not 
dependent on the existence of a small or large parameter. The method, whose sequence of solutions of linear problems convergences to the solution of the original nonlinear problem, is quadratic and monotone, which is one of the reasons for the popularity of this technique. This method was generalized by Lakshmikantham and Vatsala [10] in which the convexity or concavity assumption on the nonlinear functions involved in the problems is relaxed.

So far, the general quasilinearization method, coupled with the method of upper and lower solutions, has been applied to obtain approximation of solutions for a large number of nonlinear problems, for example, BVPs of ordinary differential equations, such as firstorder BVP with nonlinear boundary condition [11] and second-order BVPs with Dirichlet boundary condition [12], periodic boundary condition [13], three-point boundary condition [14], four-point boundary condition [15], and m-point boundary condition [16]; BVPs of partial differential equations, such as parabolic initial-boundary value problem [17], elliptic problems with nonlinear boundary condition [18] and $p$-Laplacian equations with nonlinear boundary condition [19], and so forth; BVPs of impulsive differential equations [20] and impulse functional differential equations with anti-periodic boundary condition [21]; BVPs of some practically nonlinear problems, such as Duffing equation involving both integral and nonintegral forcing terms with Robin boundary condition [22] and forced Duffing equation with discontinuous-type integral boundary condition [23]; as well as some abstract problems such as fixed point theorems in ordered Banach space [24].

Recently, El-Gebeily and O'Regan [25,26] consider the singular and nonsingular second-order ordinary differential equations

$$
l(u(t))=f(t, u(t)), \quad t \in(a, b)
$$

with nonlinear boundary conditions as follows:

$$
\begin{aligned}
& g\left(u(a), u(b), p u^{\prime}(a)\right)=0 \\
& h\left(u(a), u(b), p u^{\prime}(b)\right)=0
\end{aligned}
$$

in which $l u=-\left(p u^{\prime}\right)^{\prime}+q u$ is a formally self-adjoint second-order differential operator. This type of BVPs arises in a variety of problems in applied mathematics and physics [27]. By defining the upper and lower solutions of BVP (1.1)-(1.3) suitably, Gebeily and Regan established the existence and uniqueness of solutions and constructed two monotonic iterative sequences converging to the unique solution quadratically. However, it can be noted that the $u^{\prime}(b)$ and $u^{\prime}(a)$ terms are not involved in (1.2) and (1.3), respectively. For secondorder two-point BVPs, if all the terms $u(a), u(b), u^{\prime}(a)$ and $u^{\prime}(b)$ are involved in the boundary conditions, it seems quite difficult to deal with.

In this paper, we consider a second-order BVP with nonlinear and mixed two-point boundary conditions as follows:

$$
\begin{gathered}
L x=f\left(t, x, x^{\prime}\right), \quad t \in(a, b), \\
g\left(x(a), x(b), x^{\prime}(a), x^{\prime}(b)\right)=0, \\
x(b)=x(a),
\end{gathered}
$$

where $L x=-\left(p x^{\prime}\right)^{\prime}+q x$, in which $p \in C^{1}[a, b], p>0$, and $q \in C[a, b], q \geq 0$. 
In BVP (1.4)-(1.6), it can be found that the boundary condition (1.5) is dependent on all the $x(a), x(b), x^{\prime}(a)$, and $x^{\prime}(b)$ terms. First, existence and uniqueness of solutions of BVP (1.4)-(1.6) is established by combining the method of upper and lower solutions with LeraySchauder degree theory. Then, the general quasilinearization method is applied to construct the approximations of the unique solution. Two monotone sequences of iterations converging to the unique solution quadratically are obtained.

\section{Preliminaries}

In this section, several definitions and lemmas needed to the main results are given first.

Definition 2.1. $\beta(t), \alpha(t) \in C^{2}[a, b]$ are called the upper and lower solutions of BVP (1.4)-(1.6), respectively, if

$$
\begin{gathered}
L \beta \geq f\left(t, \beta, \beta^{\prime}\right), \quad t \in(a, b), \\
g\left(\beta(a), \beta(b), \beta^{\prime}(a), \beta^{\prime}(b)\right) \leq 0, \\
\beta(b)=\beta(a), \\
L \alpha \leq f\left(t, \alpha, \alpha^{\prime}\right), \quad t \in(a, b), \\
g\left(\alpha(a), \alpha(b), \alpha^{\prime}(a), \alpha^{\prime}(b)\right) \geq 0, \\
\alpha(b)=\alpha(a) .
\end{gathered}
$$

Definition 2.2. Let $E$ be a subset of $[a, b] \times R^{2}$; it is said that the right-hand side function of (1.4) satisfies Nagumo condition on $E$ if

$$
\left|f\left(t, x, x^{\prime}\right)\right| \leq h\left(\left|x^{\prime}\right|\right)=\mathrm{O}\left(\left|x^{\prime}\right|^{2}\right)
$$

holds for $\left(t, x, x^{\prime}\right) \in E$ and $\left|x^{\prime}\right| \rightarrow+\infty$.

Lemma 2.3 (see [28]). Let $f:[a, b] \times R^{2} \rightarrow R$ be a continuous function satisfying Nagumo condition on

$$
E=\left\{\left(t, x, x^{\prime}\right) \in[a, b] \times R^{2}: \alpha(t) \leq x(t) \leq \beta(t)\right\}
$$

where $\alpha, \beta:[a, b] \rightarrow R$ are continuous functions such that $\alpha(t) \leq \beta(t)$ for all $t \in[a, b]$. Then there exists a constant $N>0$ such that every solution $x(t)$ of second-order equations $x^{\prime \prime}=f\left(t, x, x^{\prime}\right)$ with

$$
\alpha(t) \leq x(t) \leq \beta(t), \quad t \in[a, b]
$$

satisfies $\left\|x^{\prime}\right\|_{\infty} \leq N$, in which $N$ is called the Nagumo constant. 
Lemma 2.4. Boundary value problem as follows:

$$
\begin{aligned}
& L x=-x, \quad t \in(a, b), \\
& x(a)=0, \quad x(b)=0
\end{aligned}
$$

has only the trivial solution.

Proof. Assume that $x_{0}(t)$ is an arbitrarily nontrivial solution of BVP (2.5)-(2.6). From the boundary conditions (2.6), it can be concluded that $x_{0}(t)$ can achieve its positive maximum or negative minimum in the interior of $[a, b]$, suppose at $t_{0}, t_{0} \in(a, b)$.

If $x_{0}(t)$ achieves its positive maximum, then

$$
x_{0}\left(t_{0}\right)>0, \quad x_{0}^{\prime}\left(t_{0}\right)=0, \quad x_{0}^{\prime \prime}\left(t_{0}\right) \leq 0
$$

which means that

$$
L x_{0}\left(t_{0}\right)=-p\left(t_{0}\right) x_{0}^{\prime \prime}\left(t_{0}\right)-p^{\prime}\left(t_{0}\right) x_{0}^{\prime}\left(t_{0}\right)+q\left(t_{0}\right) x\left(t_{0}\right) \geq 0 \text {. }
$$

On the other hand, it can be derived from (2.5) that

$$
L x_{0}\left(t_{0}\right)=-x_{0}\left(t_{0}\right)<0 \text {. }
$$

It is a contradiction.

If $x_{0}(t)$ achieves its negative minimum, similar arguments lead to a contradiction too. Hence, BVP (2.5)-(2.6) has only the trivial solution.

Lemma 2.5 (see [26]). Define a linear operator

$$
l: C^{1}[a, b] \longrightarrow C[a, b] \times R \times R
$$

by

$$
l x(t)=\left(p x^{\prime}(a)-p x^{\prime}(t)+\int_{a}^{t} q(s) x(s) d s, x(a), x(b)\right) .
$$

Then $l^{-1}$ exists and is continuous.

Lemma 2.6. Assume that

(1) $\alpha(t), \beta(t)$ are the lower and upper solutions of BVP (1.4)-(1.6), respectively;

(2) $f\left(t, x, x^{\prime}\right)$ is continuous on $[a, b] \times R^{2}$ and is strictly decreasing in $x$ on

$$
D=\left\{\left(t, x, x^{\prime}\right) \in[a, b] \times R^{2}: \min \{\alpha(t), \beta(t)\} \leq x \leq \max \{\alpha(t), \beta(t)\}\right\}
$$


(3) $g$ is continuously differentiable on $R^{4}$, strictly decreasing in the first and second variables, and nondecreasing and nonincreasing, respectively, in the third and forth variables.

Then,

$$
\alpha(t) \leq \beta(t), \quad t \in[a, b]
$$

Proof. Suppose that $\alpha(t)>\beta(t)$ for some $t \in[a, b]$. Then there exist some $t_{0} \in[a, b]$ such that

$$
\max _{t \in[a, b]}[\alpha(t)-\beta(t)]=\alpha\left(t_{0}\right)-\beta\left(t_{0}\right)>0 .
$$

If $t_{0} \in(a, b)$, then $\alpha\left(t_{0}\right)>\beta\left(t_{0}\right), \alpha^{\prime}\left(t_{0}\right)=\beta^{\prime}\left(t_{0}\right)$, and $\alpha^{\prime \prime}\left(t_{0}\right) \leq \beta^{\prime \prime}\left(t_{0}\right)$ and consequently,

$$
L\left[\alpha\left(t_{0}\right)-\beta\left(t_{0}\right)\right]=L \alpha\left(t_{0}\right)-L \beta\left(t_{0}\right) \geq 0
$$

However, it follows from Definition 2.1 and mean value theorem that

$$
\begin{aligned}
L \alpha\left(t_{0}\right)-L \beta\left(t_{0}\right) & \leq f\left(t_{0}, \alpha\left(t_{0}\right), \alpha^{\prime}\left(t_{0}\right)\right)-f\left(t_{0}, \beta\left(t_{0}\right), \beta^{\prime}\left(t_{0}\right)\right) \\
& =f_{x}^{\prime}\left(t_{0}, \xi, \beta^{\prime}\left(t_{0}\right)\right)\left(\alpha\left(t_{0}\right)-\beta\left(t_{0}\right)\right) \\
& <0
\end{aligned}
$$

in which $\xi \in\left(\beta\left(t_{0}\right), \alpha\left(t_{0}\right)\right)$, and the last inequality follows from the strictly decreasing property of $f$ in $x$. It is a contradiction.

If $t_{0}=a$, then $\alpha(a)>\beta(a)$ and

$$
\alpha^{\prime}(a)-\beta^{\prime}(a)=\lim _{t \rightarrow a^{+}} \frac{[\alpha(t)-\beta(t)]-[\alpha(a)-\beta(a)]}{t-a} \leq 0 .
$$

By the definitions of the lower and upper solutions, we have

$$
\alpha(b)=\alpha(a)>\beta(a)=\beta(b) .
$$

Moreover,

$$
\alpha^{\prime}(b)-\beta^{\prime}(b)=\lim _{t \rightarrow b^{-}} \frac{(\alpha(t)-\beta(t))-(\alpha(b)-\beta(b))}{t-b}=\lim _{t \rightarrow b^{-}} \frac{(\alpha(t)-\beta(t))-(\alpha(a)-\beta(a))}{t-b} \geq 0 .
$$

Consequently, in view of the monotonicity of $g$ in its variables, it follows that

$$
0 \geq g\left(\beta(a), \beta(b), \beta^{\prime}(a), \beta^{\prime}(b)\right)>g\left(\alpha(a), \alpha(b), \alpha^{\prime}(a), \alpha^{\prime}(b)\right) \geq 0,
$$

which is a contradiction.

If $t_{0}=b$, similar deductions lead to a contradiction too. Hence, Lemma 2.6 is proved. 


\section{Existence and Uniqueness of Solutions}

Theorem 3.1. Assume that

(1) $\alpha(t), \beta(t)$ are the lower and upper solutions of BVP (1.4)-(1.6), respectively;

(2) $f\left(t, x, x^{\prime}\right)$ is continuous on $[a, b] \times R^{2}$ and is strictly decreasing in $x$ on

$$
D=\left\{\left(t, x, x^{\prime}\right) \in[a, b] \times R^{2}: \alpha(t) \leq x(t) \leq \beta(t)\right\}
$$

and satisfies Nagumo condition on D;

(3) $g$ is continuously differentiable, strictly decreasing in the first and second variables, nondecreasing and nonincreasing, respectively, in the third and forth variables on $[\alpha(a), \beta(a)] \times[\alpha(b), \beta(b)] \times R^{2}$.

Then there exists a unique solution $x(t) \in C^{2}[a . b]$ of BVP (1.4)-(1.6) such that

$$
\alpha(t) \leq x(t) \leq \beta(t), \quad t \in[a, b] .
$$

Proof. Define

$$
w(t, x)= \begin{cases}\alpha(t), & x(t)<\alpha(t) \\ x(t), & \alpha(t) \leq x(t) \leq \beta(t), \\ \beta(t), & x(t)>\beta(t) .\end{cases}
$$

Introduce the following auxiliary BVPs with homotopy character:

$$
\begin{gathered}
\operatorname{Lx}=\lambda f\left(t, w(t, x), x^{\prime}\right)-x+\lambda w(t, x), \\
x(a)=\lambda\left[g\left(w(a, x(a)), w(b, x(b)), x^{\prime}(a), x^{\prime}(b)\right)+w(a, x(a))\right], \\
x(b)=x(a)
\end{gathered}
$$

in which $\lambda \in[0,1]$ is called the embedded parameter.

By the continuity of $f$ and $g$, and by the boundedness of $\alpha(t)$ and $\beta(t)$, we can select a sufficiently large constant $M_{0}>0$ such that

$$
\begin{gathered}
-M_{0}<\alpha(t) \leq \beta(t)<M_{0}, \\
f(t, \beta(t), 0)+\beta(t)<M_{0}, \\
g(\beta(a), \beta(b), 0,0)+\beta(a)<M_{0}, \\
-M_{0}<f(t, \alpha(t), 0)+\alpha(t), \\
-M_{0}<g(\alpha(a), \alpha(b), 0,0)+\alpha(a) .
\end{gathered}
$$

In what follows, the proof of the existence of solutions is divided into four steps. 
Step 1. Show that, for $\lambda \in[0,1]$, every solution $x(t)$ of BVPs (3.4) satisfies

$$
|x(t)|<M_{0}, \quad t \in[a, b] .
$$

Suppose that the estimate $|x(t)|<M_{0}$ is not true. Then, there must be some points in $[a, b]$ such that either $x(t) \geq M_{0}$ or $x(t) \leq-M_{0}$. For the former case, $x(t)$ has a positive maximum, suppose at $t_{1}$, that is,

$$
\max _{t \in[a, b]} x(t)=x\left(t_{1}\right)\left(\geq M_{0}>0\right)
$$

can be assumed. We have three cases to consider.

Case $1\left(t_{1} \in(a, b)\right)$. In this case, $x\left(t_{1}\right) \geq M_{0}>\beta\left(t_{1}\right), x^{\prime}\left(t_{1}\right)=0$ and $x^{\prime \prime}\left(t_{1}\right) \leq 0$ which leads to $L x\left(t_{1}\right) \geq 0$. On the other hand, since the definition of $w(t, x)$ yields $w\left(t_{1}, x\left(t_{1}\right)\right)=\beta\left(t_{1}\right)$; hence, for $\lambda \in(0,1]$, we have

$$
\begin{aligned}
\operatorname{Lx}\left(t_{1}\right) & =\lambda f\left(t_{1}, \beta\left(t_{1}\right), 0\right)-x\left(t_{1}\right)+\lambda \beta\left(t_{1}\right) \\
& =\lambda\left[f\left(t_{1}, \beta\left(t_{1}\right), 0\right)+\beta\left(t_{1}\right)\right]-x\left(t_{1}\right) \\
& \leq \lambda\left[f\left(t_{1}, \beta\left(t_{1}\right), 0\right)+\beta\left(t_{1}\right)-M_{0}\right]<0,
\end{aligned}
$$

in which the last inequality is obtained by the inequality (3.6). It is a contradiction.

For $\mathcal{\lambda}=0$, it can be derived that

$$
0 \leq L x\left(t_{1}\right)=-x\left(t_{1}\right) \leq-M_{0}<0
$$

which is also a contradiction.

Case $2\left(t_{1}=a\right)$. In this case, $x(a) \geq M_{0}>\beta(a), x^{\prime}(a) \leq 0$. Furthermore, we have

$$
\begin{gathered}
x(b)=x(a) \geq M_{0}>\beta(b), \\
x^{\prime}(b)=\lim _{t \rightarrow b^{-}} \frac{x(t)-x(b)}{t-b}=\lim _{t \rightarrow b^{-}} \frac{x(t)-x(a)}{t-b} \geq 0 .
\end{gathered}
$$

The definition of $w(t, x)$ means that $w(a, x(a))=\beta(a)$ and $w(b, x(b))=\beta(b)$. Hence, for $\lambda \in(0,1]$, in view of $x^{\prime}(a) \leq 0$ and $x^{\prime}(b) \geq 0$, it follows from the monotonicity of $g$ in its third and forth variables and the inequality (3.7) that

$$
\begin{aligned}
M_{0} \leq x(a) & =\lambda\left[g\left(w(a, x(a)), w(b, x(b)), x^{\prime}(a), x^{\prime}(b)\right)+w(a, x(a))\right] \\
& =\lambda\left[\beta(a)+g\left(\beta(a), \beta(b), x^{\prime}(a), x^{\prime}(b)\right)\right] \\
& \leq \lambda[\beta(a)+g(\beta(a), \beta(b), 0,0)] \\
& <\lambda M_{0} \leq M_{0}
\end{aligned}
$$

which is a contradiction. 
For $\lambda=0$, we can obtain from the second equation in (3.4) that

$$
0<M_{0} \leq x(a)=0,
$$

which is a contradiction too.

Case $3\left(t_{1}=b\right)$. In this case, $x(b) \geq M_{0}>\beta(b), x^{\prime}(b) \geq 0$, and furthermore,

$$
\begin{gathered}
x(a)=x(b) \geq M_{0}>\beta(a), \\
x^{\prime}(a)=\lim _{t \rightarrow a^{+}} \frac{x(t)-x(a)}{t-a}=\lim _{t \rightarrow a^{+}} \frac{x(t)-x(b)}{t-a} \leq 0 .
\end{gathered}
$$

The same deductions with those in Case 2 yield, for $\lambda \in(0,1]$, that

$$
\begin{aligned}
M_{0} \leq x(b)=x(a) & \leq \lambda[\beta(a)+g(\beta(a), \beta(b), 0,0)] \\
& <\lambda M_{0} \leq M_{0} .
\end{aligned}
$$

It is also a contradiction. For $\lambda=0$, it can be deduced from the boundary conditions of (3.4) that

$$
0<M_{0} \leq x(b)=x(a)=0
$$

which is a contradiction too.

Step 2. Show that there exists a positive constant $M_{1}$ such that, for $\lambda \in[0,1]$, every solution $x(t)$ of BVPs (3.4) satisfies

$$
\left|x^{\prime}(t)\right|<M_{1}, \quad t \in[a, b]
$$

Let

$$
E=\left\{\left(t, x, x^{\prime}\right) \in[a, b] \times R^{2}:|x(t)|<M_{0}\right\}
$$

Define

$$
F_{\lambda}\left(t, x, x^{\prime}\right)=\frac{1}{-p}\left[p^{\prime} x^{\prime}-q x+\lambda f\left(t, w(t, x), x^{\prime}\right)-x+\lambda w(t, x)\right], \quad\left(t, x, x^{\prime}\right) \in E
$$

Consequently, (1.4) can be rewritten as

$$
x^{\prime \prime}=F_{\lambda}\left(t, x, x^{\prime}\right)
$$


Since the function $f$ satisfies Nagumo condition on $E$,

$$
\begin{aligned}
\left|F_{\lambda}\left(t, x, x^{\prime}\right)\right| & =\frac{1}{p}\left|p^{\prime} x^{\prime}-q x+\lambda f\left(t, w(t, x), x^{\prime}\right)-x+\lambda w(t, x)\right| \\
& =\mathrm{O}\left(\left|x^{\prime}\right|^{2}\right), \quad \text { for }\left(t, x, x^{\prime}\right) \in E,\left|x^{\prime}\right| \longrightarrow+\infty
\end{aligned}
$$

is obvious if the boundedness of $x(t)$ and $w(t, x), p \in C^{1}[a, b], p>0$, and $q \in C[a, b]$ is kept in mind. That is, $F_{\lambda}\left(t, x, x^{\prime}\right)$ satisfies Nagumo condition on $E$. Hence, by Lemma 2.3, the estimate $\left|x^{\prime}(t)\right|<M_{1}, t \in[a, b]$, can be obtained.

Step 3. Show that for $\lambda=1$, BVP (3.4) has at least one solution $x_{1}(t)$.

Define a nonlinear operator

$$
N_{\lambda}: C^{1}[a, b] \longrightarrow C[a, b] \times R \times R
$$

by

$$
N_{\lambda} x(t)=\left(\int_{a}^{t}\left[\lambda f\left(s, w(s, x), x^{\prime}\right)-x+\lambda w(s, x)\right] d s, A_{\lambda}, B_{\lambda}\right)
$$

with

$$
\begin{gathered}
A_{\curlywedge}=\lambda\left[g\left(w(a, x(a)), w(b, x(b)), x^{\prime}(a), x^{\prime}(b)\right)+w(a, x(a))\right], \\
B_{\lambda}=x(a) .
\end{gathered}
$$

Consequently, BVPs (3.4) are equivalent to the following operator equations:

$$
\left(I-l^{-1} N_{\curlywedge}\right) x=0
$$

in which $l$ is the linear operator defined in Lemma 2.5, and $I$ is the unit operator.

Define the norm in $C[a, b] \times R \times R$ as

$$
\|x(t)\|_{C[a, b] \times R \times R}=\|x(t)\|_{C[a, b]}+\left|R_{1}\right|+\left|R_{2}\right|
$$

for $x(t) \in C[a, b] \times R \times R$, in which $R_{1}, R_{2}$ are two real numbers.

Let $\left\{x_{n}(t)\right\}$ be the bounded sequence on $C^{1}[a, b]$. It then follows from Steps 1 and 2 that $\left\{N_{\curlywedge} x_{n}\right\}$ and $\left\{N_{\lambda}^{\prime} x_{n}\right\}$ are both uniformly bounded in the sense of the norm defined above. Thus, $\left\{N_{\curlywedge} x_{n}\right\}$ is equicontinuous on $C[a, b] \times R \times R$. Consequently, Arzela-Ascoli theorem yields that $\left\{N_{\curlywedge} x_{n}\right\}$ is compact on $C[a, b] \times R \times R$. Therefore, $N_{\curlywedge} x$ is a completely continuous operator.

Furthermore, the operator

$$
T_{\curlywedge}: C^{1}[a, b] \longrightarrow C^{1}[a, b]
$$


defined by

$$
T_{\mathcal{\lambda}}=l^{-1} N_{\curlywedge}
$$

is also a completely continuous operator.

Define a bounded and open domain as follows:

$$
\Omega=\left\{x(t) \in C^{1}[a, b]:|x(t)|<M_{0},\left|x^{\prime}(t)\right|<M_{1}\right\} .
$$

It follows from Steps 1 and 2 that

$$
\left(I-l^{-1} N_{\curlywedge}\right) \partial \Omega \neq 0 .
$$

Therefore, the degree $\operatorname{Deg}\left(I-l^{-1} N_{\curlywedge}, \Omega, 0\right)$ is well defined. Then the invariance of degree under homotopy yields

$$
\operatorname{Deg}\left(I-l^{-1} N_{1}, \Omega, 0\right)=\operatorname{Deg}\left(I-l^{-1} N_{0}, \Omega, 0\right)
$$

Since the operator equation $\left(I-l^{-1} N_{0}\right) x=0$ is equivalent to BVP (2.5)-(2.6) which has only the trivial solution, therefore

$$
\operatorname{Deg}\left(I-l^{-1} N_{1}, \Omega, 0\right)=\operatorname{Deg}\left(I-l^{-1} N_{0}, \Omega, 0\right)= \pm 1
$$

Consequently, the operator equation $\left(I-l^{-1} N_{1}\right) x=0$ gives

$$
\begin{gathered}
L x=f\left(s, w(s, x), x^{\prime}\right)-x+w(s, x), \\
x(a)=g\left(w(a, x(a)), w(b, x(b)), x^{\prime}(a), x^{\prime}(b)\right)+w(a, x(a)), \\
x(b)=x(a),
\end{gathered}
$$

which has at least one solution $x_{1}(t) \in C^{2}[a, b]$.

Step 4. Show that every solution $x(t) \in C^{2}[a, b]$ of BVP (3.36) satisfies

$$
\alpha(t) \leq x(t) \leq \beta(t), \quad t \in[a, b] .
$$

The right-hand side of this inequality is first proved. For the sake of contradiction, suppose that $x(t)>\beta(t)$ for some $t \in[a, b]$. Define $h(t)=x(t)-\beta(t)$, then $h(t)$ has a positive maximum at some $t_{1} \in[a, b]$.

Case $1\left(t_{1} \in(a, b)\right)$. In this case, $h\left(t_{1}\right)=x\left(t_{1}\right)-\beta\left(t_{1}\right)>0, h^{\prime}\left(t_{1}\right)=x^{\prime}\left(t_{1}\right)-\beta^{\prime}\left(t_{1}\right)=0$, and $h^{\prime \prime}\left(t_{1}\right) \leq 0$ and consequently, $\operatorname{Lh}\left(t_{1}\right) \geq 0$. 
On the other hand,

$$
\begin{aligned}
\operatorname{Lh}\left(t_{1}\right) & =\operatorname{Lx}\left(t_{1}\right)-L \beta\left(t_{1}\right) \\
& \leq f\left(t_{1}, w\left(t_{1}, x\left(t_{1}\right)\right), x^{\prime}\left(t_{1}\right)\right)-x\left(t_{1}\right)+w\left(t, x\left(t_{1}\right)\right)-f\left(t_{1}, \beta\left(t_{1}\right), \beta^{\prime}\left(t_{1}\right)\right) \\
& =f\left(t_{1}, \beta\left(t_{1}\right), \beta^{\prime}\left(t_{1}\right)\right)-x\left(t_{1}\right)+\beta\left(t_{1}\right)-f\left(t_{1}, \beta\left(t_{1}\right), \beta^{\prime}\left(t_{1}\right)\right) \\
& =-x\left(t_{1}\right)+\beta\left(t_{1}\right)<0,
\end{aligned}
$$

which is a contradiction.

Case $2\left(t_{1}=a\right)$. In this case,

$$
h(a)=x(a)-\beta(a)>0, \quad h^{\prime}(a)=x^{\prime}(a)-\beta^{\prime}(a) \leq 0 .
$$

Furthermore, we have

$$
\begin{gathered}
\beta(b)=\beta(a)<x(a)=x(b), \\
x^{\prime}(b)-\beta^{\prime}(b)=h^{\prime}(b)=\lim _{t \rightarrow b^{-}} \frac{h(t)-h(b)}{t-b}=\lim _{t \rightarrow b^{-}} \frac{h(t)-h(a)}{t-b} \geq 0 .
\end{gathered}
$$

The definition of $w(t, x)$ yields $w(a, x(a))=\beta(a)$ and $w(b, x(b))=\beta(b)$. In view of $x^{\prime}(a) \leq$ $\beta^{\prime}(a)$ and $x^{\prime}(b) \geq \beta^{\prime}(b)$, it then follows from the monotonicity of $g$ in its third and forth variables that

$$
\begin{aligned}
\beta(a)<x(a) & =g\left(w(a, x(a)), w(b, x(b)), x^{\prime}(a), x^{\prime}(b)\right)+w(a, x(a)) \\
& \leq g\left(\beta(a), \beta(b), \beta^{\prime}(a), \beta^{\prime}(b)\right)+\beta(a) \\
& \leq \beta(a) .
\end{aligned}
$$

It is also a contradiction.

Case $3\left(t_{1}=b\right)$. In this case,

$$
h(b)=x(b)-\beta(b)>0, \quad h^{\prime}(b)=x^{\prime}(b)-\beta^{\prime}(b) \geq 0 .
$$

Furthermore,

$$
\begin{gathered}
\beta(a)=\beta(b)<x(b)=x(a), \\
x^{\prime}(a)-\beta^{\prime}(a)=h^{\prime}(a)=\lim _{t \rightarrow a^{+}} \frac{h(t)-h(a)}{t-a}=\lim _{t \rightarrow a^{+}} \frac{h(t)-h(b)}{t-a} \leq 0 .
\end{gathered}
$$

Similar deductions lead to a contradiction too.

Therefore, the inequality $x(t) \leq \beta(t)$ holds. In a similar way, $\alpha(t) \leq x(t)$ can be proved. 
Till now, by Steps $1-4$, the proof of the existence of solutions is completed. In what follows, we turn to the proof of the uniqueness of solutions.

Define

We may assume that $x_{1}(t)$ and $x_{2}(t)$ are two arbitrary solutions of BVP (1.4)-(1.6).

$$
y(t)=x_{1}(t)-x_{2}(t), \quad t \in[a, b]
$$

If $y(t) \equiv 0$ for $t \in[a, b]$, the uniqueness of solutions is obvious. Otherwise, there must be some points in $[a, b]$ such that either $y(t)>0$ or $y(t)<0$. For the former case, we define

$$
\max _{t \in[a, b]} y(t):=y\left(t_{1}\right)
$$

Similarly, we only need to consider the following three cases.

Case $1\left(t_{1} \in(a, b)\right)$. In this case,

$$
y\left(t_{1}\right)=x_{1}\left(t_{1}\right)-x_{2}\left(t_{1}\right)>0, \quad y^{\prime}\left(t_{1}\right)=x_{1}^{\prime}\left(t_{1}\right)-x_{2}^{\prime}\left(t_{1}\right)=0, \quad y^{\prime \prime}\left(t_{1}\right) \leq 0 .
$$

Thus, $0 \leq L y\left(t_{1}\right)$. On the other hand, the mean value theorem and the strictly decreasing property of $f\left(t, x, x^{\prime}\right)$ in $x$ results that

$$
\begin{aligned}
\operatorname{Ly}\left(t_{1}\right) & =L x_{1}\left(t_{1}\right)-L x_{2}\left(t_{1}\right) \\
& =f\left(t_{1}, x_{1}\left(t_{1}\right), x_{1}^{\prime}\left(t_{1}\right)\right)-f\left(t_{1}, x_{2}\left(t_{1}\right), x_{2}^{\prime}\left(t_{1}\right)\right) \\
& =f_{x}^{\prime}\left(t_{1}, \theta, x_{1}^{\prime}\left(t_{1}\right)\right)\left(x_{1}\left(t_{1}\right)-x_{2}\left(t_{1}\right)\right)<0,
\end{aligned}
$$

which is a contradiction, where $\theta \in\left(x_{2}\left(t_{1}\right), x_{1}\left(t_{1}\right)\right)$.

Case $2\left(t_{1}=a\right)$. In this case,

$$
y(a)=x_{1}(a)-x_{2}(a)>0, \quad y^{\prime}(a)=x_{1}^{\prime}(a)-x_{2}^{\prime}(a) \leq 0 .
$$

Moreover,

$$
\begin{gathered}
y(b)=x_{1}(b)-x_{2}(b)=x_{1}(a)-x_{2}(a)=y(a)>0, \\
x_{1}^{\prime}(b)-x_{2}^{\prime}(b)=y^{\prime}(b)=\lim _{t \rightarrow b^{-}} \frac{y(t)-y(b)}{t-b}=\lim _{t \rightarrow b^{-}} \frac{y(t)-y(a)}{t-b} \geq 0 .
\end{gathered}
$$

Nevertheless, in view of the monotonicity of $g$ in its variables, it follows from the mean value theorem that

$$
\begin{aligned}
0 & =g\left(x_{1}(a), x_{1}(b), x_{1}^{\prime}(a), x_{1}^{\prime}(b)\right)-g\left(x_{2}(a), x_{2}(b), x_{2}^{\prime}(a), x_{2}^{\prime}(b)\right) \\
& =g_{1}^{\prime}\left[x_{1}(a)-x_{2}(a)\right]+g_{2}^{\prime}\left[x_{1}(b)-x_{2}(b)\right]+g_{3}^{\prime}\left[x_{1}^{\prime}(a)-x_{2}^{\prime}(a)\right]+g_{4}^{\prime}\left[x_{1}^{\prime}(b)-x_{2}^{\prime}(b)\right]<0,
\end{aligned}
$$


which is a contradiction, in which $g_{i}^{\prime}=g_{i}^{\prime}\left(\xi_{1}, \xi_{2}, \xi_{3}, \xi_{4}\right), i=1,2,3,4, \xi_{1} \in\left(x_{2}(a), x_{1}(a)\right)$ and $\xi_{2}, \xi_{3}, \xi_{4}$ can be located by analogy.

Case $3\left(t_{1}=b\right)$. For this case, in the same way, it can be proved that this case is also impossible.

Consequently, the conclusion in Theorem 3.1 is proved.

\section{Approximations of the Unique Solution}

In this section, a series of boundary value problems whose sequence of solutions converging to the unique solution of BVP (1.4)-(1.6) is constructed.

Theorem 4.1. Assume that

(1) there exist the upper and lower solutions $\beta_{0}(t), \alpha_{0}(t)$ of $B V P(1.4)-(1.6)$;

(2) $f\left(t, x, x^{\prime}\right)$ is continuous on $[a, b] \times R^{2}$ and satisfies Nagumo condition on

$$
D=\left\{\left(t, x, x^{\prime}\right) \in[a, b] \times R^{2}: \alpha_{0}(t) \leq x(t) \leq \beta_{0}(t)\right\} ;
$$

(3) $f_{x x}\left(t, x, x^{\prime}\right)$ exists and is continuous on $[a, b] \times R^{2}$ and $f_{x}\left(t, x, x^{\prime}\right)<0, f_{x x}\left(t, x, x^{\prime}\right) \leq 0$ on $D \times[-C, C]$, where

$$
C \geq \max \left\{N, \max _{t \in[a, b]} \alpha_{0}^{\prime}(t), \max _{t \in[a, b]} \beta_{0}^{\prime}(t)\right\}
$$

is chosen sufficiently large, and $N$ is the Nagumo constant;

(4) $g(u, v, z, w) \in C^{1}\left(\left[\alpha_{0}(a), \beta_{0}(a)\right] \times\left[\alpha_{0}(b), \beta_{0}(b)\right] \times R^{2}, R\right)$, and it is strictly decreasing in $u$ and $v$, no-decreasing and nonincreasing, respectively, in $z$ and $w$.

Then there exist a monotone nonincreasing sequence $\left\{\beta_{n}(t)\right\}$ and a monotone nondecreasing sequence $\left\{\alpha_{n}(t)\right\}$ converging uniformly to the unique solution of BVP (1.4)-(1.6). Moreover, the rate of the convergence is quadratic.

Proof. Define a piecewise function $q\left(x^{\prime}\right)=\max \left\{-C, \min \left\{x^{\prime}, C\right\}\right\}$ for $x^{\prime} \in R$, and introduce $f\left(t, x, q\left(x^{\prime}\right)\right)$ on $D \times[-C, C]$ and

$$
F\left(t, x, x^{\prime} ; z\right)=f\left(t, z, q\left(x^{\prime}\right)\right)+f_{x}\left(t, z, q\left(x^{\prime}\right)\right)(x-z)
$$

for $\left(t, x, x^{\prime} ; z\right) \in D \times[-C, C] \times\left[\alpha_{0}(t), \beta_{0}(t)\right]$.

Obviously, $F\left(t, x, x^{\prime} ; z\right)$ is linear in $x$ if $z$ is viewed as a known quantity. Moreover, $f\left(t, x, q\left(x^{\prime}\right)\right)$ and $F\left(t, x, x^{\prime} ; z\right)$ both satisfy the Nagumo condition.

We begin by considering the following BVP:

$$
\begin{gathered}
L x=F\left(t, x, x^{\prime} ; \beta_{0}\right), \\
g\left(x(a), x(b), x^{\prime}(a), x^{\prime}(b)\right)=0, \\
x(b)=x(a) .
\end{gathered}
$$


In view of the definition of the upper solution $\beta_{0}(t)$ of BVP (1.4)-(1.6), it can be verified directly that

$$
\begin{gathered}
L \beta_{0} \geq f\left(t, \beta_{0}, \beta_{0}^{\prime}\right)=F\left(t, \beta_{0}, \beta_{0}^{\prime} ; \beta_{0}\right), \\
g\left(\beta_{0}(a), \beta_{0}(b), \beta_{0}^{\prime}(a), \beta_{0}^{\prime}(b)\right) \leq 0, \\
\beta_{0}(b)=\beta_{0}(a) .
\end{gathered}
$$

Hence, $\beta_{0}(t)$ is the upper solution of BVP (4.4).

According to condition (3), it can be deduced that

$$
\begin{aligned}
& F\left(t, \alpha_{0}, \alpha_{0}^{\prime} ; \beta_{0}\right)-f\left(t, \alpha_{0}, \alpha_{0}^{\prime}\right) \\
&= f\left(t, \beta_{0}, \alpha_{0}^{\prime}\right)+f_{x}\left(t, \beta_{0}, \alpha_{0}^{\prime}\right)\left(\alpha_{0}-\beta_{0}\right)-f\left(t, \alpha_{0}, \alpha_{0}^{\prime}\right) \\
&= f\left(t, \beta_{0}, \alpha_{0}^{\prime}\right)+f_{x}\left(t, \beta_{0}, \alpha_{0}^{\prime}\right)\left(\alpha_{0}-\beta_{0}\right) \\
&-\left[f\left(t, \beta_{0}, \alpha_{0}^{\prime}\right)+f_{x}\left(t, \beta_{0}, \alpha_{0}^{\prime}\right)\left(\alpha_{0}-\beta_{0}\right)+\frac{1}{2} f_{x x}\left(t, \xi, \alpha_{0}^{\prime}\right)\left(\alpha_{0}-\beta_{0}\right)^{2}\right] \\
&=-\frac{1}{2} f_{x x}\left(t, \xi_{1}, \alpha_{0}^{\prime}\right)\left(\alpha_{0}-\beta_{0}\right)^{2} \geq 0,
\end{aligned}
$$

in which $\left|\alpha_{0}^{\prime}\right|<C$ has been taken into account, and $\xi_{1} \in\left(\alpha_{0}, \beta_{0}\right)$. Consequently, by taking the definition of the lower solution $\alpha_{0}(t)$ of BVP (1.4)-(1.6) into account, we have

$$
\begin{gathered}
L \alpha_{0} \leq f\left(t, \alpha_{0}, \alpha_{0}^{\prime}\right) \leq F\left(t, \alpha_{0}, \alpha_{0}^{\prime} ; \beta_{0}\right) \\
g\left(\alpha_{0}(a), \alpha_{0}(b), \alpha_{0}^{\prime}(a), \alpha_{0}^{\prime}(b)\right) \geq 0, \\
\alpha_{0}(b)=\alpha_{0}(a)
\end{gathered}
$$

which indicates that $\alpha_{0}(t)$ is the lower solution of BVP (4.4). Since BVP (4.4) satisfies all the requirements in Theorem 3.1, hence BVP (4.4) has a unique solution $\beta_{1}(t) \in C^{2}[a, b]$ with $\alpha_{0}(t) \leq \beta_{1}(t) \leq \beta_{0}(t)$ and $\left|\beta_{1}^{\prime}(t)\right|<C_{1}, t \in[a, b]$, where $C_{1} \leq C$ is a constant.

Next, we consider

$$
\begin{gathered}
L x=F\left(t, x, x^{\prime} ; \beta_{1}\right), \\
g\left(x(a), x(b), x^{\prime}(a), x^{\prime}(b)\right)=0, \\
x(b)=x(a) .
\end{gathered}
$$


It can be shown that

$$
\begin{aligned}
L \beta_{1} & =F\left(t, \beta_{1}, \beta_{1}^{\prime} ; \beta_{0}\right) \\
& =f\left(t, \beta_{0}, \beta_{1}^{\prime}\right)+f_{x}\left(t, \beta_{0}, \beta_{1}^{\prime}\right)\left(\beta_{1}-\beta_{0}\right) \\
& \geq f\left(t, \beta_{0}, \beta_{1}^{\prime}\right)+f_{x}\left(t, \beta_{0}, \beta_{1}^{\prime}\right)\left(\beta_{1}-\beta_{0}\right)+\frac{1}{2} f_{x x}\left(t, \xi_{2}, \beta_{1}^{\prime}\right)\left(\beta_{1}-\beta_{0}\right)^{2} \\
& =f\left(t, \beta_{1}, \beta_{1}^{\prime}\right)=F\left(t, \beta_{1}, \beta_{1}^{\prime} ; \beta_{1}\right)
\end{aligned}
$$

in which $\xi_{2} \in\left(\beta_{1}, \beta_{0}\right)$. Thus, $\beta_{1}(t)$ is the upper solution of BVP (4.8) since

$$
\begin{gathered}
g\left(\beta_{1}(a), \beta_{1}(b), \beta_{1}^{\prime}(a), \beta_{1}^{\prime}(b)\right) \leq 0, \\
\beta_{1}(b)=\beta_{1}(a)
\end{gathered}
$$

are obvious if we keep that $\beta_{1}(t)$ is the solution of BVP (4.4) in mind.

Similarly, we can show that $\alpha_{0}(t)$ is the lower solution of BVP (4.8). Hence, according to Theorem 3.1, there exists a unique solution $\beta_{2}(t) \in C^{2}[a, b]$ of BVP (4.8) such that $\alpha_{0}(t) \leq$ $\beta_{2}(t) \leq \beta_{1}(t)$ and $\left|\beta_{2}^{\prime}(t)\right|<C_{1}, t \in[a, b]$, where $C_{1}$ is a constant.

By repeating the above procedure iteratively, we obtain a monotone nonincreasing sequence $\left\{\beta_{n}(t)\right\}$ with

$$
\alpha_{0}(t) \leq \cdots \leq \beta_{n}(t) \leq \cdots \leq \beta_{1}(t) \leq \beta_{0}(t), \quad\left|\beta_{i}^{\prime}(t)\right| \leq C_{1}, t \in[a, b]
$$

where $\left\{\beta_{n}(t)\right\}$ are, respectively, the unique solution of the BVPs as follows:

$$
\begin{gathered}
L x=F\left(t, x, x^{\prime} ; \beta_{n-1}\right), \\
g\left(x(a), x(b), x^{\prime}(a), x^{\prime}(b)\right)=0, \\
x(b)=x(a) .
\end{gathered}
$$

Formula (4.11), the properties of the operator $L$, and the continuity of $F$ yield the uniform boundedness of $\left\{\beta_{n}^{\prime \prime}(t)\right\}$ for $t \in[a, b]$. Therefore, the sequences $\left\{\beta_{n}^{i}(t)\right\}(i=0,1)$ are uniformly bounded and equicontinuous on $[a, b]$. Hence, Ascoli-Arzela theorem guarantees the existence of subsequences $\left\{\beta_{n_{k}}^{i}(t)\right\} \subset\left\{\beta_{n}^{i}(t)\right\}$ and a function $x(t) \in C^{1}[a, b]$ with $\left\{\beta_{n_{k}}^{i}(t)\right\}$ converging uniformly to $x^{i}(t)(i=0,1)$ on $[a, b]$ as $k \rightarrow+\infty$.

Now let

$$
\begin{gathered}
f_{k}=F\left(t, \beta_{n_{k}}, \beta_{n_{k}}^{\prime} ; \beta_{n_{k}-1}\right), \\
g_{k}=g\left(\beta_{n_{k}}(a), \beta_{n_{k}}(b), \beta_{n_{k}}^{\prime}(a), \beta_{n_{k}}^{\prime}(b)\right) .
\end{gathered}
$$


The continuity of the functions $F$ and $g$ imply that, as $k \rightarrow+\infty$,

$$
\begin{gathered}
f_{k}=F\left(t, \beta_{n_{k}}, \beta_{n_{k}}^{\prime} ; \beta_{n_{k}-1}\right) \longrightarrow f\left(t, x, x^{\prime}\right), \\
g_{k}=g\left(\beta_{n_{k}}(a), \beta_{n_{k}}(b), \beta_{n_{k}}^{\prime}(a), \beta_{n_{k}}^{\prime}(b)\right) \longrightarrow g\left(x(a), x(b), x^{\prime}(a), x^{\prime}(b)\right) .
\end{gathered}
$$

The Lebeague dominated convergence theorem then yields

$$
\int_{a}^{t} f_{k}(s) d s \longrightarrow \int_{a}^{t} f\left(s, x(s), x^{\prime}(s)\right) d s
$$

for each $t \in[a, b]$ as $k \rightarrow+\infty$.

Integrating the equation

$$
L \beta_{n_{k}}=F\left(t, \beta_{n_{k}}, \beta_{n_{k}}^{\prime} ; \beta_{n_{k}-1}\right)
$$

from $a$ to $t$ results in

$$
p \beta_{n_{k}}^{\prime}(a)-p \beta_{n_{k}}^{\prime}(t)+\int_{a}^{t} q(s) \beta_{n_{k}}(s) d s=\int_{a}^{t} f_{k}(s) d s .
$$

Passing to the limit in (4.17), we obtain

$$
p x^{\prime}(a)-p x^{\prime}(t)+\int_{a}^{t} q(s) x(s) d s=\int_{a}^{t} f\left(s, x(s), x^{\prime}(s)\right) d s .
$$

Hence, $x(t)$ is the unique solution of BVP (1.4)-(1.6).

To show the quadratic rate of convergence, we define the error function as follows:

$$
e_{n}(t)=\beta_{n}(t)-x(t)
$$

Hence, $e_{n}(t) \geq 0$ for $t \in[a, b]$.

$$
\begin{aligned}
L e_{n}= & L\left(\beta_{n}-x\right)=L \beta_{n}-L x \\
= & F\left(t, \beta_{n}, \beta_{n}^{\prime} ; \beta_{n-1}\right)-f\left(t, x, x^{\prime}\right) \\
= & f\left(t, \beta_{n-1}, \beta_{n}^{\prime}\right)+f_{x}\left(t, \beta_{n-1}, \beta_{n}^{\prime}\right)\left(\beta_{n}-\beta_{n-1}\right)-f\left(t, x, x^{\prime}\right) \\
= & f\left(t, x, \beta_{n}^{\prime}\right)-f\left(t, x, x^{\prime}\right)+f\left(t, \beta_{n-1}, \beta_{n}^{\prime}\right)-f\left(t, x, \beta_{n}^{\prime}\right) \\
& +f_{x}\left(t, \beta_{n-1}, \beta_{n}^{\prime}\right)\left(\beta_{n}-x\right)+f_{x}\left(t, \beta_{n-1}, \beta_{n}^{\prime}\right)\left(x-\beta_{n-1}\right) \\
= & f_{x^{\prime}}\left(t, \beta_{n-1}, \eta_{1}\right) e_{n}^{\prime}+f_{x}\left(t, \beta_{n-1}, \beta_{n}^{\prime}\right) e_{n}-\frac{1}{2} f_{x x}\left(t, \eta_{2}, \beta_{n}^{\prime}\right)\left(x-\beta_{n-1}\right)^{2} \\
\leq & f_{x^{\prime}}\left(t, \beta_{n-1}, \eta_{1}\right) e_{n}^{\prime}+K_{1} e_{n}+K_{2}\left\|e_{n-1}\right\|^{2},
\end{aligned}
$$


in which $\eta_{1}$ lies between $\beta_{n}^{\prime}$ and $x^{\prime}, \eta_{2} \in\left(x, \beta_{n-1}\right), K_{1}=\max _{t \in[a, b]} f_{x}\left(t, \beta_{n-1}, \beta_{n}^{\prime}\right)$ is a negative constant, $K_{2}$ is a constant with $0 \leq-(1 / 2) f_{x x}\left(t, \eta_{2}, \beta_{n}^{\prime}\right) \leq K_{2}$ for $\left(t, \eta_{2}, \beta_{n}^{\prime}\right) \in D \times[-C, C]$, and $\left\|e_{n-1}\right\|=\max _{t \in[a, b]}\left|e_{n-1}(t)\right|$.

Moreover, since $\beta_{n}(t), x(t)$ are the solutions of BVPs (4.12) and BVP (1.4)-(1.6), respectively, therefore, mean value theorem yields

$$
\begin{gathered}
g_{1}^{\prime} e_{n}(a)+g_{2}^{\prime} e_{n}(b)+g_{3}^{\prime} e_{n}^{\prime}(a)+g_{2}^{\prime} e_{n}^{\prime}(b)=0, \\
e_{n}(a)=e_{n}(b),
\end{gathered}
$$

in which $g_{i}^{\prime}=g_{i}^{\prime}\left(\eta_{3}, \eta_{4}, \eta_{5}, \eta_{6}\right), i=1,2,3,4$, where $\eta_{3} \in\left(x(a), \beta_{n}(a)\right)$, and so forth.

Define a constant function by

$$
W(t)=-\frac{K_{2}}{K_{1}}\left\|e_{n-1}\right\|^{2} \geq 0, \quad t \in[a, b] .
$$

Then, $W(t)$ satisfies

$$
L W \geq f_{x^{\prime}}\left(t, \beta_{n-1}, \eta_{1}\right) W^{\prime}+K_{1} W+K_{2}\left\|e_{n-1}\right\|^{2}
$$

and moreover, since $W(t)$ is a nonnegative constant function, therefore, we have $W(a) \geq$ $0, W(b) \geq 0$ and $W^{\prime}(a)=0, W^{\prime}(b)=0$. Consequently, the condition (4) guarantees that

$$
\begin{gathered}
g_{1}^{\prime} W(a)+g_{2}^{\prime} W(b)+g_{3}^{\prime} W^{\prime}(a)+g_{2}^{\prime} W^{\prime}(b) \leq 0, \\
W(a)=W(b)
\end{gathered}
$$

in which $g_{i}^{\prime}, i=1,2,3,4$, are defined in (4.21).

If $e_{n}(t) \leq W(t)$ holds uniformly for $t \in[a, b]$, the quadratic convergence of the iteration has been proved. Otherwise, there must be some points $t \in[a, b]$ such that $e_{n}(t)>W(t)$. We then introduce a function

$$
q_{n}(t)=e_{n}(t)-W(t), \quad t \in[a, b]
$$

and define

$$
\max _{t \in[a, b]} q_{n}(t):=q_{n}\left(t_{1}\right)
$$

Case $1\left(t_{1} \in(a, b)\right)$. In this case, $q_{n}\left(t_{1}\right)=e_{n}\left(t_{1}\right)-W\left(t_{1}\right)>0, q_{n}^{\prime}\left(t_{1}\right)=e_{n}^{\prime}\left(t_{1}\right)-W^{\prime}\left(t_{1}\right)=0$, and $q_{n}^{\prime \prime}\left(t_{1}\right)=e_{n}^{\prime \prime}\left(t_{1}\right)-W^{\prime \prime}\left(t_{1}\right) \leq 0$. Hence, $L q_{n}\left(t_{1}\right) \geq 0$. On the other hand, it can be deduced that

$$
\begin{aligned}
L q_{n}\left(t_{1}\right) & =L e_{n}\left(t_{1}\right)-L W\left(t_{1}\right) \\
& \leq f_{x^{\prime}}\left(t_{1}, \beta_{n-1}, \eta_{1}\right)\left(e_{n}^{\prime}\left(t_{1}\right)-W^{\prime}\left(t_{1}\right)\right)+K_{1}\left(e_{n}\left(t_{1}\right)-W\left(t_{1}\right)\right) \\
& =K_{1} q_{n}\left(t_{1}\right)<0
\end{aligned}
$$

which is a contradiction. 
Case $2\left(t_{1}=a\right)$. In this case, $q_{n}(a)>0, q_{n}^{\prime}(a) \leq 0$. Furthermore, we can obtain

$$
\begin{aligned}
& q_{n}(b)=e_{n}(b)-W(b)=e_{n}(a)-W(a)=q_{n}(a)>0, \\
& q_{n}^{\prime}(b)=\lim _{t \rightarrow b^{-}} \frac{q_{n}(t)-q_{n}(b)}{t-b}=\lim _{t \rightarrow b^{-}} \frac{q_{n}(t)-q_{n}(a)}{t-b} \geq 0 .
\end{aligned}
$$

Consequently, the monotonicity of $g$ given in the condition (4) yields

$$
g_{1}^{\prime} q_{n}(a)+g_{2}^{\prime} q_{n}(b)+g_{3}^{\prime} q_{n}^{\prime}(a)+g_{2}^{\prime} q_{n}^{\prime}(b)<0
$$

However, equation (4.21) minus inequality (4.25) gives

$$
g_{1}^{\prime} q_{n}(a)+g_{2}^{\prime} q_{n}(b)+g_{3}^{\prime} q_{n}^{\prime}(a)+g_{2}^{\prime} q_{n}^{\prime}(b) \geq 0
$$

which contradicts to (4.31).

Similarly, we can prove that Case 3 , that is, $t_{1}=b$, is also impossible.

Hence, the inequality

$$
e_{n}(t) \leq W(t)=-\frac{K_{2}}{K_{1}}\left\|e_{n-1}\right\|^{2}
$$

holds uniformly for $t \in[a, b]$, that is,

$$
\left\|e_{n}(t)\right\| \leq K\left\|e_{n-1}\right\|^{2}, \quad t \in[a, b]
$$

holds uniformly, in which $K=-K_{2} / K_{1} \geq 0$ and $\left\|e_{n}(t)\right\|=\max _{t \in[a, b]}\left|e_{n}(t)\right|$.

\section{An Example}

Consider a nonlinear BVP as follows:

$$
\begin{gathered}
-\left(\left(t^{2}+1\right) x^{\prime}\right)^{\prime}+k x=-\left(t^{2}+3\right) e^{x}-2\left(x^{\prime}\right)^{2}+t^{2}+1, \quad t \in(-1,1), \\
-x(-1)-x(1)+\frac{1}{3}\left(x^{\prime}(-1)\right)^{3}-\frac{1}{3}\left(x^{\prime}(1)\right)^{3}=0, \\
x(-1)=x(1)
\end{gathered}
$$

in which $k$ is a constant to be determined, that is,

$$
\begin{gathered}
L x=-\left(\left(t^{2}+1\right) x^{\prime}\right)^{\prime}+k x, \quad f\left(t, x, x^{\prime}\right)=-\left(t^{2}+3\right) e^{x}-2\left(x^{\prime}\right)^{2}+t^{2}+1, \\
g(x, y, z, w)=-x-y+\frac{1}{3} z^{3}-\frac{1}{3} w^{3}
\end{gathered}
$$

in this BVP. 
It can be verified that $\alpha_{0}(t)=-2$ and $\beta_{0}(t)=t^{2}$ are, respectively, the lower and upper solutions of BVP (5.1) if $k \geq\left(3-e^{2}\right) / 2 e^{2}$ is chosen. Moreover, it is easy to show that all the assumptions in Theorem 4.1 are satisfied. Therefore, by Theorem 4.1, BVP (5.1) has a unique solution and it can be approximated quadratically by two monotone sequences.

\section{Acknowledgment}

This work is supported by the National Natural Science Foundation of Fujian Province of China under Grant (no. S0650010).

\section{References}

[1] W. C. Lian, F. H. Wong, and C. C. Yeh, "On the existence of positive solutions of nonlinear second order differential equations," Proceedings of the American Mathematical Society, vol. 124, no. 4, pp. 11171126, 1996.

[2] Y. D. Shi, Q. D. Zhou, and Y. Li, "A note on a two-point boundary value problem arising from a liquid metal flow," SIAM Journal on Mathematical Analysis, vol. 28, no. 5, pp. 1086-1093, 1997.

[3] C. O. Horgan, G. Saccomandi, and I. Sgura, "A two-point boundary-value problem for the axial shear of hardening isotropic incompressible nonlinearly elastic materials," SIAM Journal on Applied Mathematics, vol. 62, no. 5, pp. 1712-1727, 2002.

[4] P. Amster and C. Rogers, "On boundary value problems in three-ion electrodiffusion," Journal of Mathematical Analysis and Applications, vol. 333, no. 1, pp. 42-51, 2007.

[5] J. B. Amara, "Sturm theory for the equation of vibrating beam," Journal of Mathematical Analysis and Applications, vol. 349, no. 1, pp. 1-9, 2009.

[6] S. N. Ha and C. R. Lee, "Numerical study for two-point boundary value problems using Green's functions," Computers E Mathematics with Applications, vol. 44, no. 12, pp. 1599-1608, 2002.

[7] H. H. Pang, M. Q. Feng, and W. G. Ge, "Existence and monotone iteration of positive solutions for a three-point boundary value problem," Applied Mathematics Letters, vol. 21, no. 7, pp. 656-661, 2008.

[8] A. S. Bataineh, M. S. M. Noorani, and I. Hashim, "Modified homotopy analysis method for solving systems of second-order BVPs," Communications in Nonlinear Science and Numerical Simulation, vol. 14, no. 2, pp. 430-442, 2009.

[9] R. Bellman and R. E. Kalaba, Quasilinearization and Nonlinear Boundary Value Problems, Elsevier, New York, NY, USA, 1965.

[10] V. Lakshmikantham and A. S. Vatsala, Generalized Quasilinearization for Nonlinear Problems, vol. 440 of Mathematics and Its Applications, Kluwer Academic Publishers, Dordrecht, The Netherlands, 1998.

[11] T. Jankowski, "Quadratic approximation of solutions for differential equations with nonlinear boundary conditions," Computers \& Mathematics with Applications, vol. 47, no. 10-11, pp. 1619-1626, 2004.

[12] P. W. Eloe and Y. Z. Zhang, "A quadratic monotone iteration scheme for two-point boundary value problems for ordinary differential equations," Nonlinear Analysis. Theory, Methods E Applications, vol. 33, no. 5, pp. 443-453, 1998.

[13] R. N. Mohapatra, K. Vajravelu, and Y. Yin, "Generalized quasilinearization method for second-order boundary value problems," Nonlinear Analysis. Theory, Methods E Applications, vol. 36, no. 6, pp. 799806, 1999.

[14] M. H. Pei and S. K. Chang, "The generalized quasilinearization method for second-order three-point boundary value problems," Nonlinear Analysis. Theory, Methods \& Applications, vol. 68, no. 9, pp. 27792790, 2008.

[15] M. H. Pei and S. K. Chang, "A quasilinearization method for second-order four-point boundary value problems," Applied Mathematics and Computation, vol. 202, no. 1, pp. 54-66, 2008.

[16] R. A. Khan, "Generalized approximations and rapid convergence of solutions of $m$-point boundary value problems," Applied Mathematics and Computation, vol. 188, no. 2, pp. 1878-1890, 2007.

[17] J. Yang and A. S. Vatsala, "Numerical investigation of generalized quasilinearization method for reaction diffusion systems," Computers \& Mathematics with Applications, vol. 50, no. 3-4, pp. 587-598, 2005. 
[18] P. Amster and P. De Nápoli, "A quasilinearization method for elliptic problems with a nonlinear boundary condition," Nonlinear Analysis. Theory, Methods E Applications, vol. 66, no. 10, pp. 2255-2263, 2007.

[19] G. A. Afrouzi and M. Alizadeh, "A quasilinearization method for $p$-Laplacian equations with a nonlinear boundary condition," Nonlinear Analysis. Theory, Methods \& Applications, vol. 71, no. 7-8, pp. 2829-2833, 2009.

[20] D. D. Bainov and S. G. Hristova, "The method of quasilinearization for the periodic boundary value problem for systems of impulsive differential equations," Applied Mathematics and Computation, vol. 117, no. 1, pp. 73-85, 2001.

[21] B. Ahmad and J. Nieto, "Existence and approximation of solutions for a class of nonlinear impulsive functional differential equations with anti-periodic boundary conditions," Nonlinear Analysis. Theory, Methods E Applications, vol. 69, no. 10, pp. 3291-3298, 2008.

[22] B. Ahmad and B. Alghamdi, "Approximation of solutions of the nonlinear Duffing equation involving both integral and non-integral forcing terms with separated boundary conditions," Computer Physics Communications, vol. 179, pp. 409-416, 2008.

[23] B. Ahmad and A. Alsaedi, "Existence of approximate solutions of the forced Duffing equation with discontinuous type integral boundary conditions," Nonlinear Analysis. Real World Applications, vol. 10, no. 1, pp. 358-367, 2009.

[24] V. Lakshmikantham, S. Carl, and S. Heikkilä, "Fixed point theorems in ordered Banach spaces via quasilinearization," Nonlinear Analysis. Theory, Methods \& Applications, vol. 71, no. 7-8, pp. 3448-3458, 2009.

[25] M. El-Gebeily and D. O'Regan, “A quasilinearization method for a class of second order singular nonlinear differential equations with nonlinear boundary conditions," Nonlinear Analysis. Real World Applications, vol. 8, no. 1, pp. 174-186, 2007.

[26] M. El-Gebeily and D. O'Regan, "A generalized quasilinearization method for second-order nonlinear differential equations with nonlinear boundary conditions," Journal of Computational and Applied Mathematics, vol. 192, no. 2, pp. 270-281, 2006.

[27] R. Ma, R. Wang, and L. Ren, "Existence results for semipositone boundary value problems," Acta Mathematica Scientia. Series B, vol. 21, no. 2, pp. 189-195, 2001.

[28] M. R. Grossinho and F. M. Minhós, "Upper and lower solutions for higher order boundary value problems," Nonlinear Studies, vol. 12, no. 2, pp. 165-176, 2005. 


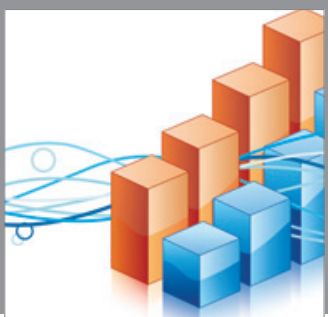

Advances in

Operations Research

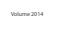

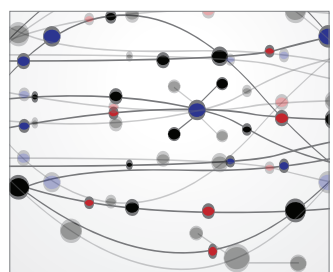

\section{The Scientific} World Journal
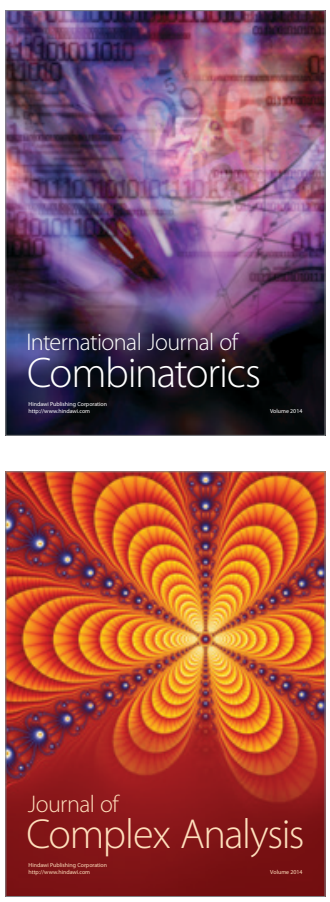

International Journal of

Mathematics and

Mathematical

Sciences
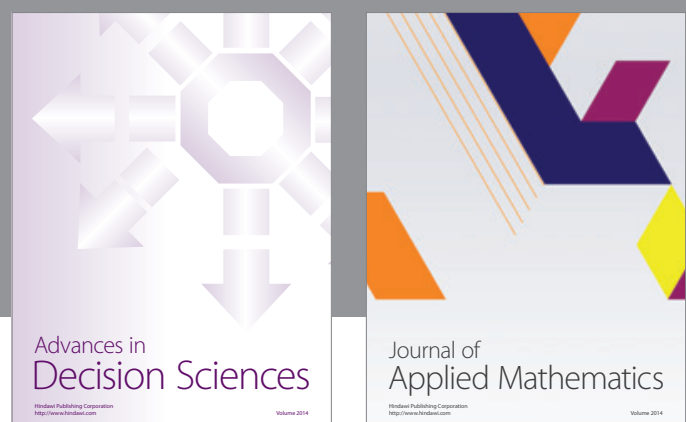

Journal of

Applied Mathematics
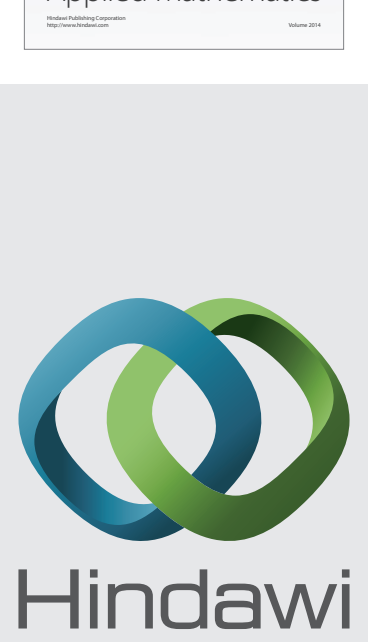

Submit your manuscripts at http://www.hindawi.com
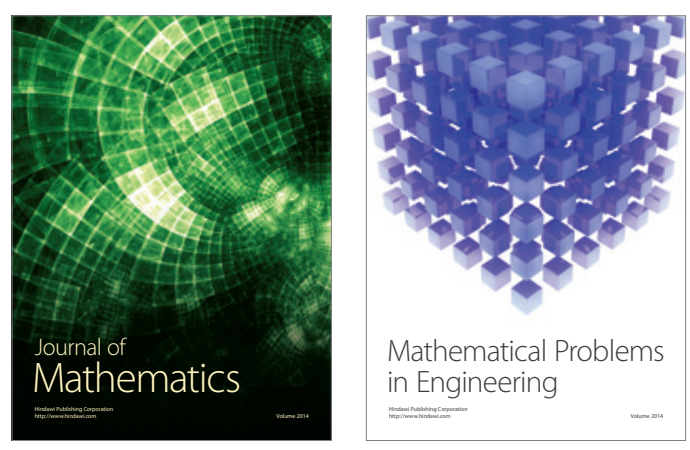

Mathematical Problems in Engineering
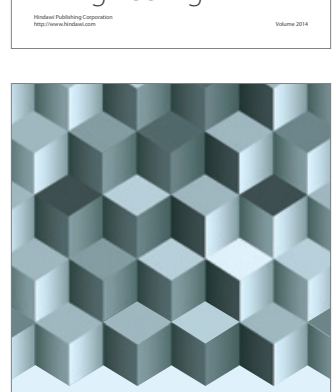

Journal of

Function Spaces
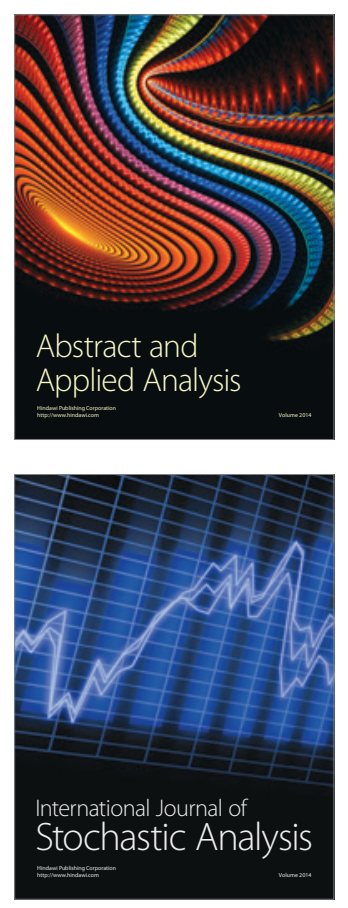

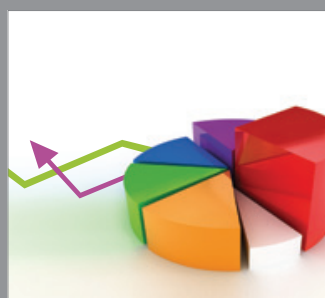

ournal of

Probability and Statistics

Promensencen
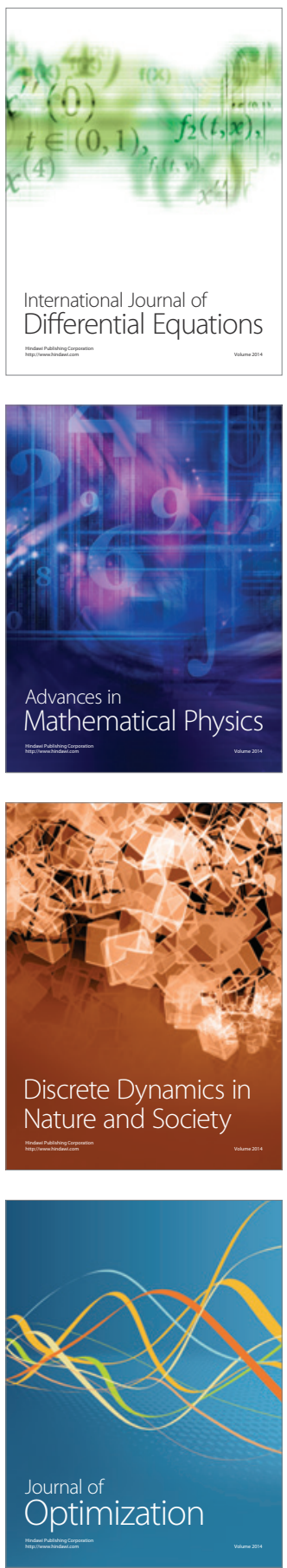